\title{
Stochastic Modeling Framework for Wireless Power Transfer in the Radiative Near-Field
}

\author{
Thomas Deckmyn, Marco Rossi, Dries Vande Ginste, Hendrik Rogier \\ Ghent University-imec: Dept. of Information Technology, Technologiepark Zwijnaarde 15, \\ 9052 Ghent, Belgium, thomas.deckmyn@ugent.be
}

\begin{abstract}
Given the growing interest in wireless power transfer, there is a need to quickly evaluate the efficiency of such links for different power source and receiver separations and orientations. Moreover, a designer is not only interested in the nominal operating conditions, but also in the variations encountered due to variability and uncertainty in the system parameters and deployment conditions. We therefore propose a stochastic modeling framework to evaluate the efficiency of a wireless power transfer system in both the radiative near-field and far-field. Nominal performance and variations in the figures of merit of the system are rapidly accounted for by leveraging a vector spherical wave expansion, Wigner-D rotations and a stochastic testing procedure.
\end{abstract}

Index Terms - wireless power transfer, wireless channel modeling, stochastic testing.

\section{INTRODUCTION}

The interest in wireless power transfer (WPT) has increased explosively the last years, fueled by recent evolutions such as the Internet of Things and Electrical Vehicles. It is clear that, for the safe transfer of large power levels, inductive powering via the near-field is to be preferred. However, a large plethora of applications exist where huge amounts of wireless sensors nodes need to be powered wirelessly at rather low power levels. These nodes are typically extremely difficult to access, distributed over large areas and deployed in huge quantities. The only option to recharge them is via WPT links that sweep over the coverage area in a rather quick manner, without paying particular attention to each individual node.

Still, although it is impossible to provide individual care to each node separately, the contribution of every sensor is crucial to maintaining detailed coverage of the area to be monitored. Therefore, there is a particular need for a modeling tool that evaluates not only the nominal power transfer efficiency (PTE) of a WPT system, but that also studies all potential random variations and uncertainties due to random system tolerances and receiver/transmitter locations. Moreover, WPT proves to be more efficient at smaller distances. Therefore, besides being based on the conventional far-field approximations, such a modeling framework must also be able to accurately predict the PTE in radiative near-field conditions.

In this contribution, we present a novel modeling paradigm to evaluate the nominal PTE and its statistical variations for links in the Fresnel and Fraunhofer regions. The transmit and receive antenna systems are described by commercial electromagnetic (EM) field simulators. These component models are complemented by a hybrid plane-wave/vector-sphericalwave expansion based on a Green-function addition theorem to model the radiowave propagation characteristics down to distances of a few wavelengths and a generalized polynomial chaos expansion (gPC) to include variability and uncertainty. Once the radiation patterns of the different antennas are known, the framework allows to model repositioning and reorientation of the transmit and receive systems without requiring any full-wave simulation. This, in turn, yields efficient uncertainty and variability modeling for varying deployment conditions and random fabrication errors.

In Section II, we describe the deterministic model that first creates component models for the power source with the transmit antenna and the receive rectenna. We introduce the wireless channel model that accurately describes far-field as well as radiative near-field power transfer between the transmit and receive antennas. It is based on a plane-wave expansion of the Green function through an addition theorem. This yields a description for the interaction between the radiation patterns of transmit and receive antenna by a translation operator, which is easily and accurately calculated for any set of antenna positions in the radiative near-field. Next, the radiation patterns are expanded into vector-spherical modes, enabling easy rotation of both antennas through Wigner-D matrices. In Section III, we introduce the stochastic framework, which operates around the deterministic model in a non-intrusive manner. To obtain the probability density function of the PTE, instead of performing Monte Carlo simulations, which only converge with the inverse of the square root of the number of processed realizations [1], in Section IV we leverage gPC expansions [2], [3], to assess the impact of antenna variability and positioning uncertainties on the PTE. Given the large number of random variables present in the complete wireless link, we introduce Stochastic Testing [4], [5] to reduce the number of deterministic simulations needed to gather the statistical data. In Section V, the approach is validated on a simple WPT system consisting of a transmitting horn antenna and a receiving Industrial, Scientific and Medical (ISM) flexible antenna operating at $2.45 \mathrm{GHz}$.

\section{Modeling the nOMinal PTE}

Consider a WPT system whose building blocks are schematically shown in Fig. 1. Note that, although in the scheme a single transmit and receive antenna are assumed, the scheme 


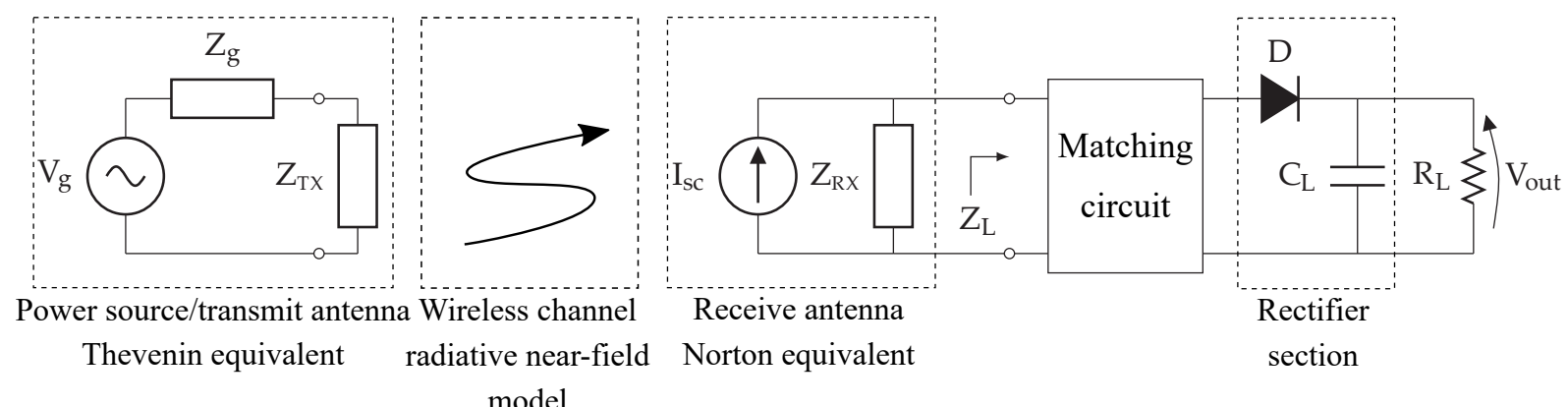

Fig. 1. Building blocks of the WPT system model for evaluation of the power transfer efficiency.

can be extended to antenna arrays at both sides of the link [6] On the one hand, the power source is represented by its Thevenin equivalent, connected to a transmit antenna with input impedance $Z_{T X}$. On the other hand, the receive antenna is described by its Norton equivalent, composed of the antenna input impedance $Z_{R X}$ and its short-circuit current source $I_{S C}$, related to the incoming field by

$$
I_{S C}=-\frac{1}{C} \int_{\Omega} T\left(\mathbf{r}_{R X}, \mathbf{r}_{T X}, \hat{k}\right) \mathbf{F}_{R X}(\hat{k}) \cdot \mathbf{F}_{T X}(\hat{k}) d \hat{k},
$$

where the integration extends over the Ewald sphere $\Omega$, with $C$ a normalization constant, $\mathbf{F}(\hat{k})$ the radiation pattern of transmit or receive antenna, and the translation operator given by

$$
\begin{aligned}
& T\left(\mathbf{r}_{R X}, \mathbf{r}_{T X}, \hat{k}\right)= \\
& \quad \sum_{n=0}^{N}(2 n+1) j^{-n} h_{n}^{(2)}\left(k r_{R X, T X}\right) P_{n}\left(\hat{k} \cdot \hat{r}_{R X, T X}\right),
\end{aligned}
$$

with $r_{R X, T X}=\left|\mathbf{r}_{R X}-\mathbf{r}_{T X}\right|$ and $\hat{r}_{R X, T X}=\frac{\mathbf{r}_{R X}-\mathbf{r}_{T X}}{r_{R X, T X}}$. $h_{n}^{(2)}(\cdot)$ denotes the spherical Hankel function of the second kind and order $n$, and $P_{n}(\cdot)$ represents the Legendre polynomial of degree $n$. The relative positions of the transmit and receive antenna can be easily modified without requiring fullwave simulations, since the antenna radiation patterns remain unchanged. Only (2) must be updated to account for the new positions. Moreover, the distance between transmit and receive antenna may be reduced, bringing both antennas in each other's radiative near field, at the cost of more terms (larger order $N$ ) in (2). To reorient both antennas without performing any EM field simulation, we expand their radiation patterns into vector spherical harmonics and apply Wigner-D rotations to these expansion coefficients. After reassembling both patterns, we can evaluate (1) in a straightforward manner, given these reoriented radiation patterns. For all mathematical details about this model, we refer to [7].

\section{INCLUDING VARIABILITY AND UNCERTAINTY}

During WPT, variability in the antenna dimensions and uncertainty in deployment conditions, modifying the antenna positions and orientations, will affect the PTE in a random manner. To quantify the statistical distribution resulting from all random variables in the link, we leverage a a generalized polynomial chaos expansion of the form

$$
\mathrm{PTE} \approx \sum_{\boldsymbol{l}=0}^{L} y_{\boldsymbol{l}}^{\boldsymbol{x}} \boldsymbol{\phi}_{\boldsymbol{l}}^{\boldsymbol{x}}(\boldsymbol{x}) .
$$

with $\boldsymbol{x}$ a vector containing all input random variables, related to the PTE through a multivariate polynomial of degree $L$. Prior to this expansion, the random variables $x_{k}$ in the vector have been made statistically independent through a Choleski decomposition. An exponentially converging expansion is obtained when and orthogonal set of expansion polynomials $\phi_{l}^{\boldsymbol{x}}(\boldsymbol{x})$ is chosen, with orthogonality defined by

$$
\int_{\Omega_{k}} \phi_{i, k}^{x_{k}}\left(x_{k}\right) \phi_{j, k}^{x_{k}}\left(x_{k}\right) d \mathcal{P}^{x_{k}}=\left|\phi_{i, k}^{x_{k}}\left(x_{k}\right)\right|^{2} \delta_{i j} .
$$

In order to perform the stochastic analysis without requiring the modify the deterministic approach outlined in Section II, we apply the statistical framework in a non-intrusive manner. To calculate $y_{k}^{X}$ in expansion (3), we exploit (4) and obtain

$$
\begin{aligned}
y_{\boldsymbol{l}}^{\boldsymbol{x}} & =E\left[\mathrm{PTE}(\boldsymbol{x}) \boldsymbol{\phi}_{\boldsymbol{l}}^{\boldsymbol{x}}(\boldsymbol{x})\right] \\
& =\int_{\Omega_{1}, \ldots, \Omega_{L}} \operatorname{PTE}(\boldsymbol{x}) \phi_{l_{1}, 1}^{x_{1}}\left(x_{1}\right) \ldots \phi_{l_{K}, K}^{x_{K}}\left(x_{K}\right) d \mathcal{P}^{x_{1}} \ldots d \mathcal{P}^{x_{K}} .
\end{aligned}
$$

This integral may be evaluated by a multidimensional Gauss quadrature rule, such as a tensor product rule or a Stroud cubature [8]. Yet, for a large number of random variables, this approach requires an excessively large number of WPT link realizations. The Stochastic Testing algorithm [4], [9] drastically reduces the number of tensor product Gaussian quadrature points needed to construct the probability density function of the PTE. First, the initial set of quadrature points is sorted according to their corresponding quadrature weights. Then, after selecting the point with the most dominant weight, of the remaining points, only those are chosen that significantly contribute to the quadrature rule via a thresholding procedure outlined in [4].

\section{STOCHASTIC WPT LINK MODEL}

We now outline the stochastic framework to quantify the variability and uncertainty in the WPT setup shown in Fig. 1. 
The nominal PTE is evaluated according to the method outlined in Section II, for each radiative near-field link realization at given transmit and receive antenna positions and orientations. The rectifier network of the received antenna is analyzed by a harmonic balance simulation in Keysight's Advanced Design System to obtain PTE $=\eta_{\text {link }} \cdot \eta_{\text {match }} \cdot \eta_{\text {rect }}$ as a product of the WPT link efficiency $\eta_{\text {link }}$, matching network efficiency $\eta_{\text {match }}$ and rectifier efficiency $\eta_{\text {rect }}$.

\section{A. Accounting for antenna variability}

To simplify the analysis, in this contribution we will assume that only the receive antenna is subject to variability. Variations in the transmit antenna may be included in the framework by a straightforward extension of the theory. Random changes in antenna performance may be due to uncertain antenna dimensions and variations in relative permittivity $\epsilon_{r}$. Their effects on the radiation impedance $Z_{\mathrm{RX}}=Z^{r e}+j Z^{i m}$ and radiation pattern are described by polynomial chaos expansions, given by

$$
\begin{aligned}
Z^{r e, i m} & =\sum_{\boldsymbol{l}_{1}=0}^{L_{Z^{r e, i m}}} y_{\boldsymbol{l}_{1}}^{\boldsymbol{x}} \boldsymbol{\phi}_{\boldsymbol{l}_{1}}^{\boldsymbol{x}}\left(\mathbf{x}^{\mathrm{VAR}}\right) \\
A_{p q}^{r e, i m} & =\sum_{A_{p q}^{r e, i m}}^{\boldsymbol{l}_{2}=0} y_{\boldsymbol{l}_{2}}^{\boldsymbol{x}} \boldsymbol{\phi}_{\boldsymbol{l}_{2}}^{\boldsymbol{x}}\left(\mathbf{x}^{\mathrm{VAR}}\right) \\
B_{p q}^{r e, i m} & =\sum_{\boldsymbol{B}_{3}=0}^{L_{p q}^{r e, i m}} y_{\boldsymbol{l}_{3}}^{\boldsymbol{x}} \boldsymbol{\phi}_{\boldsymbol{l}_{3}}^{\boldsymbol{x}}\left(\mathbf{x}^{\mathrm{VAR}}\right),
\end{aligned}
$$

with $\mathbf{x}^{\mathrm{VAR}}$ the vector of (statistically independent) random variables related to the antenna variability, and $A_{p q}=A_{p q}^{r e}+j A_{p q}^{i m}$ and $B_{p q}=B_{p q}^{r e}+j B_{p q}^{i m}$ the spherical harmonics expansion coefficients of the antenna's radiation pattern. The latter are exploited to perform the antenna translations and rotations and evaluate the receive antenna circuits parameters in Fig. 1.

\section{B. Evaluating antenna position and orientation uncertainty}

The final gPC expansion of the overall WPT system PTE includes both antenna variability and uncertainty in position and orientation, accounted for by

$$
\mathrm{PTE}=\sum_{\boldsymbol{l}=0}^{L} y_{\boldsymbol{l}}^{\boldsymbol{x}} \boldsymbol{\phi}_{\boldsymbol{l}}^{\boldsymbol{x}}\left(\mathbf{x}^{\mathrm{WPT}}\right)
$$

with $\mathbf{x}^{\text {WPT }}$ now a vector including all random variables in the link. First, we determine (9) in $M_{\mathrm{PTE}}$ stochastic testing points $\mathbf{x}_{m}^{\mathrm{WPT}}$. For each link realization corresponding to a quadrature point, we exploit the expansions (6)-(8) constructed in Section IV-A to describe both the antenna radiation impedances and their radiation patterns. Second, application of the WPT model of Section II yields the position and orientation uncertainties $\mathbf{x}_{m}^{\mathrm{WPT}}$ in the WPT link. Finally, the PTE is evaluated for each WPT link realization. This multi-stage approach limits the number of EM field simulations needed to construct the antenna macromodels. In addition, these macromodels are reused when antenna elements in the link are deployed at different positions.

\section{EXAMPLE}

Consider a WPT link between a power source connected to an MI-212-1.72.45 GHz horn antenna, which emits $10 \mathrm{~mW}$ of power at $2.45 \mathrm{GHz}$ to a flexible dual-polarized rectenna, as shown in Fig. 2. In nominal conditions, the WPT system operates at a distance $d=0.6 \mathrm{~m} \approx 5 \lambda$ (being in the radiative near field), and both antennas are located in the $x y$-plane with their phase centers aligned. The receive antenna [10] has a nominal radiation impedance $Z_{\mathrm{RX}}=49.91-1.93 i \Omega$ at each feed, with more than $15 \mathrm{~dB}$ of isolation better the feeds. On this antenna, a rectifier, voltage doubler and matching network are deployed (Fig. 3) to generate DC power. The receive antenna suffers from variability in its dimensions and substrate properties. The most significant design parameters $L, W$ and $\epsilon_{r}$ (Fig. 2 for more details) are found [11], [12] to vary as independent Gaussian random variables with standard deviations, $\boldsymbol{\sigma}_{L}=0.1268 \mathrm{~mm}, \boldsymbol{\sigma}_{L}=0.1628 \mathrm{~mm}$ and $\sigma_{\epsilon_{r}}=0.03190$, respectively. Moreover, there is uncertainty on antenna positions $d, x, y$ and orientation angles $\theta$ and $\phi$. All again vary as Gaussian random variables with standard deviations $\sigma_{d}=16.66 \mathrm{~mm}, \sigma_{y}=\sigma_{x}=6.66 \mathrm{~mm}$ and $\boldsymbol{\sigma}_{\phi}=\boldsymbol{\sigma}_{\theta}=10^{\circ}$.

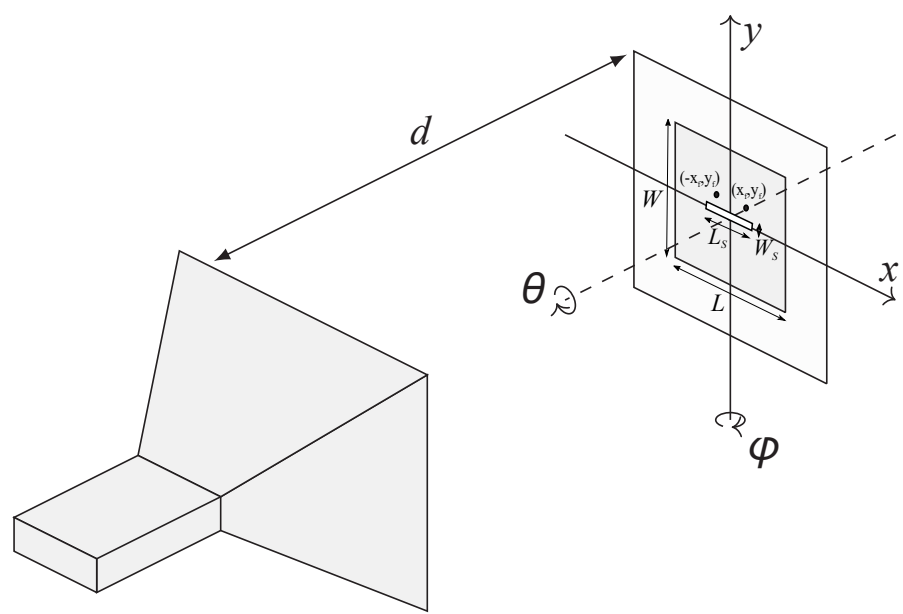

Fig. 2. WPT link between standard gain horn power source and flexible (substrate thickness $3.94 \mathrm{~mm}, \epsilon_{r}=1.5259$ ) dual-polarized rectenna at $2.45 \mathrm{GHz} . \quad L=45.3854 \mathrm{~mm}, W=44.4516 \mathrm{~mm}, L_{S}=14.88 \mathrm{~mm}$, $W_{S}=1 \mathrm{~mm},( \pm)\left(x_{f}, y_{f}\right)=( \pm 5.7,5.7) \mathrm{mm}$.

To account for the effect of all normal random variables, we construct the polynomial chaos expansions (6), (7), (8) and (9) using Hermite polynomials. The stochastic testing procedure constructs polynomial expansions (6)-(8) of degree 6 by performing 84 ADS-Momentum full-wave simulations of receive antenna realizations. Next, we concentrate on the polynomial expansion (9) in eight random variables. To obtain an expansion of degree $L=4$, stochastic testing requires 495 quadrature points $\mathbf{x}_{m}^{\mathrm{LINK}}$. The PTE is evaluated very efficiently by leveraging the approach outlined in Section II. Next, a Monte Carlo analysis based on 10000 realizations is performed to validate the stochastic WPT link model. For both approaches, the cumulative distribution function (CDF) is shown in Fig. 4. A Kolmogorov-Smirnov test confirms that 


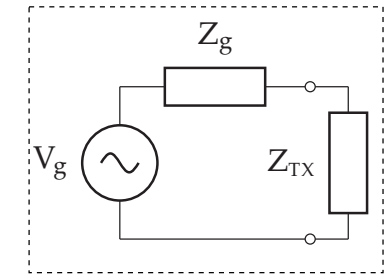

Generator+standard gain horn Thevenin equivalent

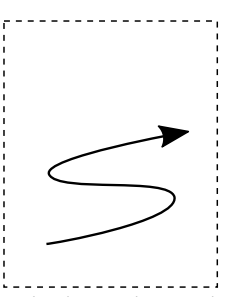

Wireless channel

radiative near-field model

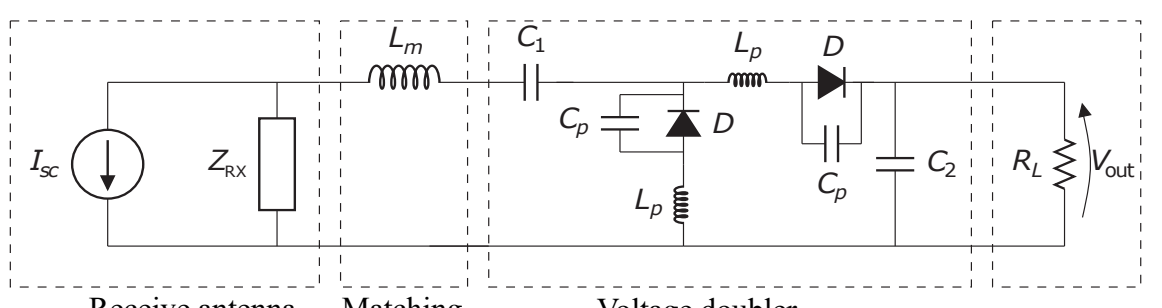

Receive antenna Matching

Norton equivalent circuit
Voltage doubler

and rectifier section

Fig. 3. Schematic of the example WPT link. $L_{m}=5 \mathrm{nH}$, diodes are HSMS-2850 Schottky diodes with $C_{p}=0.08 \mathrm{pF}$ and $L_{p}=2 \mathrm{nH}, C_{1}=C_{2}=100 \mathrm{pF}$, $R_{L}=100 \Omega$.

both curves indeed belong to the same distribution, with a significance level $\alpha=5 \%$. In the complete stochastic modeling framework, the electromagnetic field simulations turn out to be the most costly step, since one ADS Momentum full-wave antenna simulation costs $15 \mathrm{~s}$ of CPU-time, requiring a total of $21 \mathrm{~min}$ to generate the expansions (6)-(8). By leveraging these results, only an additional $54 \mathrm{~s}$ are needed to construct the final PTE expansion (9), resulting in a total CPU time of $22 \mathrm{~min}$ for the full model. In contrast, a straightforward application of (9), based on direct full-wave simulations without use of the efficient model outlined in Section II, would require $2 \mathrm{~h}$ of CPU time whereas the Monte Carlo approach needs $41 \mathrm{~h}$ to evaluate 10000 realizations.

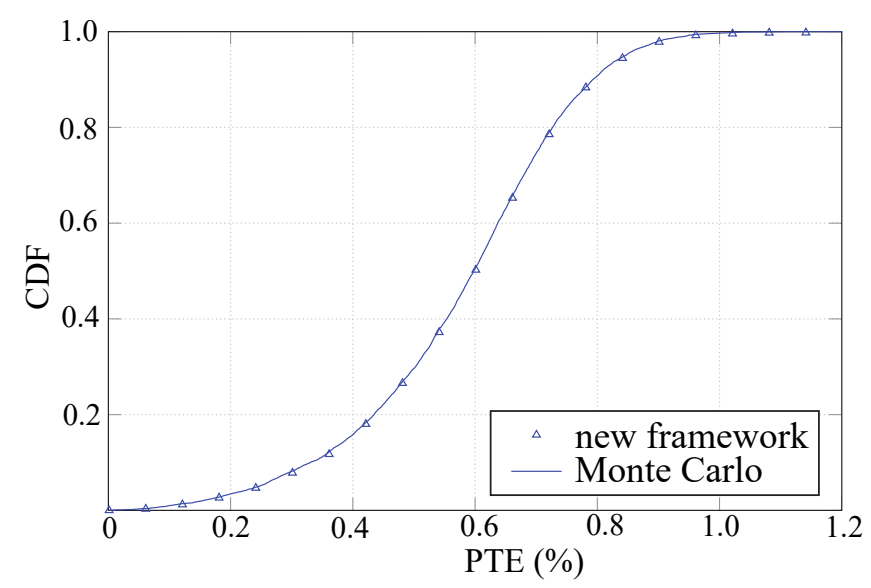

Fig. 4. CDFs of the power transfer efficiency (PTE) of the WPT link: Monte Carlo simulation versus the novel stochastic framework.

\section{CONCLUSION}

We have presented a new modeling framework to evaluate the power transfer efficiency of a WPT link in the radiative near field for both nominal and randomly varying operating conditions. The technique cleverly exploits an addition theorem for the Green function, vector spherical harmonics and Wigner-D rotations to describe the WPT channel for arbitrary positions and orientations of the transmit and receive antennas. This is combined with a stochastic testing procedure to derive the statistical distribution of the PTE from the distributions of the random variables characterizing the WPT link.

\section{ACKNOWLEDGMENT}

This research was partially funded by BELSPO and by the Research Foundation Flanders (FWO-Vlaanderen).

\section{REFERENCES}

[1] G. Fishman, Monte Carlo: concepts, algorithms, and applications. Springer Science \& Business Media, 2013.

[2] D. Xiu and G. E. Karniadakis, "The wiener-askey polynomial chaos for stochastic differential equations," SIAM Journal on Scientific Computing, vol. 24, no. 2, pp. 619-644, 2002.

[3] D. Xiu, "Fast numerical methods for stochastic computations: a review," Communications in computational physics, vol. 5, no. 2-4, pp. 242-272, 2009.

[4] Z. Zhang, T. El-Moselhy, I. Elfadel, and L. Daniel, "Stochastic testing method for transistor-level uncertainty quantification based on generalized polynomial chaos," Computer-Aided Design of Integrated Circuits and Systems, IEEE Transactions on, vol. 32, no. 10, pp. 1533-1545, 2013.

[5] P. Manfredi, D. Vande Ginste, D. De Zutter, and F. Canavero, "Generalized decoupled polynomial chaos for nonlinear circuits with many random parameters," Microwave and Wireless Components Letters, IEEE, vol. 25, no. 8, pp. 505-507, 2015.

[6] G.-J. Stockman, S. Lemey, H. Rogier, and D. Vande Ginste, "FullWave Modeling of Interacting Multiport Devices With Arbitrary Relative Positions and Orientations for Efficient EMI Assessment," IEEE Trans. on Electromagnetic Compatibility, vol. 58, no. 4, 2, SI, pp. 1322-1330, AUG 2016.

[7] G. J. Stockman, H. Rogier, and D. Vande Ginste, "Efficient modeling of interactions between radiating devices with arbitrary relative positions and orientations," IEEE Transactions on Electromagnetic Compatibility, vol. 56, no. 6, pp. 1313-1321, Dec 2014.

[8] D. Xiu and J. S. Hesthaven, "High-order collocation methods for differential equations with random inputs," SIAM Journal on Scientific Computing, vol. 27, no. 3, pp. 1118-1139, 2005.

[9] M. Rossi, G.-J. Stockman, H. Rogier, and D. Vande Ginste, "Stochastic analysis of the efficiency of a wireless power transfer system subject to antenna variability and position uncertainties," Sensors, vol. 16, no. 7, 2016.

[10] L. Vallozzi, H. Rogier, and C. Hertleer, "Dual polarized textile patch antenna for integration into protective garments," IEEE Antennas and Wireless Propagation Letters, , vol. 7, pp. 440-443, May 2008.

[11] M. Rossi, A. Dierck, H. Rogier, and D. Vande Ginste, "A stochastic framework for the variability analysis of textile antennas," Antennas and Propagation, IEEE Transactions on, vol. 62, no. 12, pp. 6510-6514, 2014.

[12] M. Rossi, S. Agneessens, H. Rogier, and D. Vande Ginste, "Stochastic analysis of the impact of substrate compression on the performance of textile antennas," IEEE Transactions on Antennas and Propagation, vol. 64, no. 6, pp. 2507-2512, June 2016. 